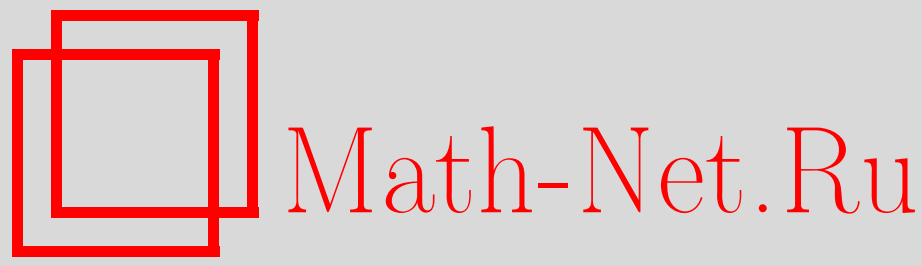

Ф. Магри, Многообразия Хаантьеса и системы Веселова, TMФ, 2016, том 189, номер 1, 101-114

DOI: https://doi.org/10.4213/tmf9077

Использование Общероссийского математического портала Math-Net.Ru подразумевает, что вы прочитали и согласны с пользовательским соглашением http: //www . mathnet.ru/rus/agreement

Параметры загрузки:

IP: 54.147 .182 .235

26 апреля 2023 г., 13:33:38

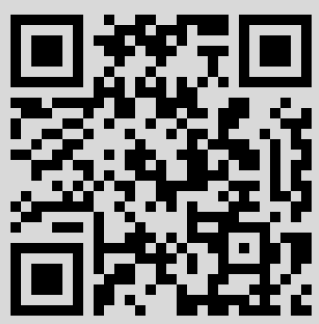


Том 189, № 1

октябрь, 2016

(C) 2016 г.

Ф. Магри*

\title{
МНОГООБРАЗИЯ ХААНТЬЕСА И СИСТЕМЫ ВЕСЕЛОВА
}

\begin{abstract}
Предложена новая геометрическая интерпретация решений уравнений Виттена-Дийкграфа-Верлинде-Верлинде, найденных ранее А. П. Веселовым.
\end{abstract}

Ключевые слова: уравнения Виттена-Дийкграфа-Верлинде-Верлинде, V-системы, комплексы Ленарда.

DOI: $10.4213 /$ tmf9077

\section{1. ВВЕДЕНИЕ}

В 1999 г. Веселов [1] нашел класс специальных решений уравнений Виттена-Дийкграфа-Верлинде-Верлинде (ВДВВ), которые играют важную роль в двумерной топологической теории поля и в $N=2$ суперсимметричной теории Янга-Милса. Эти решения связаны со специальными наборами ковекторов в линейном пространстве, называемом V-системой [1], [2]. Характерным примером этого класса решений является функция

$$
F\left(x_{1}, \ldots, x_{n}\right)=\sum_{i<j}^{n}\left(x_{i}-x_{j}\right)^{2} \ln \left(x_{i}-x_{j}\right)^{2}+\frac{1}{m} \sum_{i=1}^{n} x_{i}^{2} \ln x_{i}^{2},
$$

зависящая от произвольного параметра $m$. Соответствующая V-система является деформацией системы корней $A_{n}$.

Совсем недавно автор предложил рассмотреть специальные конфигурации 1-форм на многообразии, называемые комплексами Ленарда [3]. В настоящей статье утверждается, что V-система Веселова может быть связана с определенным классом комплексов Ленарда, которые называются эквивариантными комплексами.

Утверждение разбивается на четыре шага. В разделе 2 мы напоминаем определение комплексов Ленарда и приведены два примера. В разделе 3 объясняется связь между решениями уравнений ВДВВ и комплексами Ленарда. Один комплекс такого типа можно связать с решением уравнений ВДВВ и наоборот. В разделе 4 выводится понятие эквивариантного комплекса Ленарда. В разделе 5 мы изучаем конкретный класс эквивариантных комплексов, которые связаны с V-системами Веселова.

${ }^{*}$ Dipartimento di Matematica e Applicazioni, Universita di Milano-Bicocca, Milano, Italy. E-mail: franco.magri@unimib.it 


\section{2. КОМПЛЕКСЫ ЛЕНАРДА}

Комплекс Ленарда представляет собой составную геометрическую структуру, которая может быть определена над специальным классом многообразий, называемых многообразиями Хаантьеса. Его можно рассматривать как умеренное обобщение цепей Ленарда, первоначально определенных на бигамильтоновых многообразиях.

Чтобы определить комплекс Ленарда на многообразии $M$, нам понадобятся векторное поле $X: M \rightarrow T M$, точная 1-форма $d A: M \rightarrow T^{*} M$ и семейство тензорных полей типа $K_{j}: T M \rightarrow T M \quad(1,1)$ в количестве, совпадающем с размерностью многообразия. Согласно общепринятой терминологии эти тензорные поля мы будем называть операторами рекурсии комплекса. Мы предполагаем, что они попарно коммутируют:

$$
K_{j} K_{l}-K_{l} K_{j}=0 .
$$

Их действие на $X$ и $d A$ приводит к обычным цепям векторных полей

$$
X_{j}=K_{j} X
$$

и 1 -форм

$$
\theta_{j}=K_{j} d A
$$

Что еще более важно, они также приводят к (симметрическому) квадрату 1-форм

$$
\theta_{j l}=K_{j} K_{l} d A
$$

Этот квадрат 1-форм является главной новинкой в теории комплексов Ленарда по отношению к старой теории цепей Ленарда. Другим отличием является то, что операторы рекурсии $K_{j}$ не являются степенями одного оператора $K$. Еще одним отличием является то, что ничего не предполагается априори о кручении операторов рекурсии $K_{j}$. Они могут иметь кручение. Несмотря на это, можно показать, что операторы рекурсии комплекса Ленарда не имеют кручения Хаантьеса. Это основная причина, по которой мы выбрали многообразия Хаантьеса в качестве многообразий, допускающих структуру комплекса Ленарда.

ОпРедЕЛЕниЕ 1. Составная структура, состоящая из цепей векторных полей $X_{j}$, цепей 1-форм $\theta_{j}$ и квадрата 1-форм $\theta_{j l}$, является комплексом Ленарда на многообразии Хаантвеса $M$, если выполняются равенства

$$
\begin{aligned}
{\left[X_{j}, X_{l}\right] } & =0, \\
d \theta_{j} & =0, \\
d \theta_{j l} & =0,
\end{aligned}
$$

т. е. если векторные поля коммутируют и 1-формы являются замкнутыми, а следовательно, локально-точными.

Если, кроме того, векторные поля $X_{j}$ и 1-формы $\theta_{l}$ линейно независимы (на некотором открытом плотном подмножестве $M$ ), то из предыдущих предположений следует, что комплекс Ленарда определяет две различные системы координат на начальном многообразии $M$. 
ОПРЕДЕЛЕНиЕ 2 . Координаты $x_{j}$ и $a_{j}$, локально определенные соотношениями

$$
K_{j} X=\frac{\partial}{\partial x_{j}}, \quad K_{j} d A=d a_{j},
$$

называются $x$-координатами и $a$-координатами, индуиированными комплексом Ленарда на многообразии Хаантвеса $M$.

В дополнение к координатам $x_{j}$ и $a_{j}$ полезно также учитывать след локальных потенциалов замкнутых 1-форм $\theta_{j l}$.

ОПРЕДЕЛЕНиЕ 3. Симметричная матрица, элементами которой являются локальные потенциалы $A_{j l}$ 1-форм квадрата Ленарда

$$
K_{j} K_{l} d A=d A_{j l},
$$

называется матричным потенциалом комплекса Ленарда.

Прежде чем перейти к уравнениям ВДВВ, обсудим два примера. Они проливают свет на то, как возникают комплексы Ленарда в теории интегрируемых систем.

ПримеР 1. Рассмотрим бигамильтоново векторное поле

$$
X=P_{1} d A=P_{2} d B
$$

на бигамильтоновом многообразии $M$. Если один из пуассоновых бивекторов, скажем $P_{2}$, обратим, то с полем $X$ можно связать оператор рекурсии

$$
K=P_{1} \circ P_{2}^{-1} \text {. }
$$

Давно известно, что тройка $(K, d A, X)$ удовлетворяет соотношениям

$$
\begin{aligned}
\operatorname{Torsion}(K) & =0, \\
\operatorname{Lie}_{X}(K) & =0, \\
d(K d A) & =0,
\end{aligned}
$$

следующим из условия совместимости пуассоновых бивекторов. Кроме того, известно, что эти свойства выполняются и для степеней $K^{i}$ оператора рекурсии $K$ и что векторные поля $K^{j} X, 1$-формы $K^{i} d A$ и 1-формы $K^{j} K^{l} d A=K^{j+l} d A$ удовлетворяют условиям, определяющим комплекс Ленарда. Таким образом, комплекс Ленарда специального типа связан с любым бигамильтоновым векторным полем на симплектическом многообразии. В некотором смысле можно сказать, что концепция комплексов Ленарда берет свое начало в геометрии бигамильтоновых многообразий. Тем не менее такая постановка носит слишком ограничительный характер. Из следующего примера видно, почему удобно расширить старую схему цепей Ленарда.

ПримеР 2. Система дифференциальных уравнений в частных производных

$$
\begin{aligned}
\frac{\partial w_{2}}{\partial t} & =2 \frac{\partial w_{1}}{\partial x}, \\
\frac{\partial w_{1}}{\partial t} & =2 \frac{\partial w_{0}}{\partial x}-w_{2} \frac{\partial w_{2}}{\partial x}, \\
\frac{\partial w_{0}}{\partial t} & =-\frac{1}{2} w_{1} \frac{\partial w_{2}}{\partial x}
\end{aligned}
$$


- это классический пример интегрируемой системы гидродинамического типа. Она является представителем широкого класса интегрируемых уравнений, известных как бездисперсионные уравнения Гельфанда-Дикого. Такие уравнения интенсивно изучались последние тридцать лет, и в настоящее время известно, что они имеют много замечательных свойств, некоторые из которых достаточно сложны и глубоко скрыты (см., например, [4]). Добавим одно свойство, которое демонстрирует, что комплекс Ленарда связан с бездисперсионными уравнениями Гельфанда-Дикого.

Это стандартная практика - записать приведенные выше уравнения в матричной форме, чтобы вывести матрицу коэффициентов

$$
V=\left(\begin{array}{ccc}
0 & 2 & 0 \\
-w_{2} & 0 & 2 \\
-\frac{1}{2} w_{1} & 0 & 0
\end{array}\right)
$$

собственные значения которой являются характеристическими скоростями системы. Тем не менее эта матрица зависит от выбора координат, а следовательно, лучше использовать геометрическое представление. Чтобы преодолеть эту трудность, лучше всего преобразовать матрицу $V$ в оператор рекурсии $K$, используя условия

$$
\begin{aligned}
& K d w_{2}=2 d w_{1}, \\
& K d w_{1}=2 d w_{0}-w_{2} d w_{2}, \\
& K d w_{0}=-\frac{1}{2} w_{1} d w_{2}
\end{aligned}
$$

на некотором открытом подмножестве $U$ в $\mathbb{R}^{3}$, где $\left(w_{0}, w_{1}, w_{2}\right)$ играют роль координат, а $\left(d w_{0}, d w_{1}, d w_{2}\right)$ - соответствующий канонический базис в $T^{*} U$. Этот оператор - первая характеристика геометрического представления уравнений Гельфанда-Дикого. Другие две следуют из изучения $K$. Одна из них связана с кручением Нийенхейса $K$. Есть много способов записать это кручение, но наиболее удобная запись состоит в том, чтобы сократить векторнозначную 2-форму $\operatorname{Torsion}(K)$ с дифференциалом функции $F$ и получить скалярнозначную 2-форму $d F(\operatorname{Torsion}(K))$. В случае уравнений Гельфанда-Дикого скалярнозначная 2-форма имеет очень простой вид:

$$
d F(\operatorname{Torsion}(K))=d w_{2} \wedge d F .
$$

Эта формула показывает, что кручение оператора $K$ не может обращаться в нуль и что оно полностью характеризуется 1-формой

$$
d A=d w_{2} .
$$

Данная форма является второй интересной характеристикой геометрического представления уравнений Гельфанда-Дикого. Третья характеристика - это векторное поле

$$
X=\frac{\partial}{\partial w_{0}},
$$

которое явно является симметрией $K$. Таким образом, тройка объектов $(K, d A, X)$ естественным образом связана с данными уравнениями, как и в бигамильтоновом случае. Главным различием, однако, является то, что у оператора $K$ есть кручение. 
Соответственно, нельзя использовать “правило степеней", чтобы построить две цепи коммутирующих векторов и точных 1-форм. Существование кручения у оператора $K$ обязывает нас сделать более тонкую рекуррентную процедуру. Правильный ответ заключается в выборе коммутирующих операторов

$$
K_{1}=\mathrm{Id}, \quad K_{2}=K, \quad K_{3}=K^{2}+A \cdot \mathrm{Id} .
$$

Эти операторы, 1-форма $d A$ и векторное поле $X$ удовлетворяют условиям, определяющим комплекс Ленарда. Существование такого комплекса является еще одним проявлением интегрируемости уравнений Гельфанда-Дикого.

\section{3. УРАВНЕНИЯ ВДВВ}

Существует прямая связь между комплексами Ленарда и уравнениями ВДВВ. Она основана на сплетении квадрата 1-форм $d A_{j l}$ с $x$-координатами комплекса Ленарда. Потенциалы $A_{j l}$ квадрата 1-форм являются элементами симметричной матрицы, которую мы называем матричным потенциалом комплекса. Запишем функции $A_{j l}$ в $x$-координатах.

ПреДЛОЖЕНИЕ 1. Матричный потенииал комплекса Ленарда, записанный в $x$-координатах, есть матрица Гессе функиии F, которая удовлетворяет уравнениям ВДВВ.

ДокАЗАтельство. Вычислим частные производные функций $A_{j l}$ :

$$
\frac{\partial A_{j l}}{\partial x_{m}}=d A_{j l}\left(\frac{\partial}{\partial x^{m}}\right)=d A\left(K_{j} K_{l} K_{m} X\right) .
$$

Правая часть симметрична по индексам $(j, l, m)$, а следовательно, функции $A_{j l}$ являются элементами матрицы Гессе:

$$
A_{j l}=\frac{\partial^{2} F}{\partial x_{j} \partial x_{l}} .
$$

Чтобы показать, что потенциал $F$ удовлетворяет уравнениям ВДВВ, предположим (как это делается в рамках теории ВДВВ), что 1-формы $d A_{j l}$ при $j=1$ линейно независимы, а следовательно, образуют базис в $T^{*} M$. Это предположение гарантирует, что частные производные $\partial h / \partial x_{1}$ матрицы Гессе функции $F$

$$
h=\operatorname{Hessian}(F)
$$

обратимы. Также это гарантирует, что оператор рекурсии $K_{1}$ обратим (эти два факта, очевидным образом, связаны). Рассмотрим операторы $Q_{j}=K_{j} \circ K_{1}^{-1}$. Заметим, что

$$
Q_{j} d A_{1 l}=d A_{j l} .
$$

Тогда матрица для $Q_{j}$ в базисе $d x_{j}$ имеет вид

$$
Q_{j}=\left(\frac{\partial h}{\partial x_{1}}\right)^{-1} \frac{\partial h}{\partial x_{j}} .
$$


Так как операторы $Q_{j}$ коммутируют, то же справедливо для их матриц. Поэтому уравнение ВДВВ имеет вид

$$
\frac{\partial h}{\partial x_{j}}\left(\frac{\partial h}{\partial x_{1}}\right)^{-1} \frac{\partial h}{\partial x_{l}}=\frac{\partial h}{\partial x_{l}}\left(\frac{\partial h}{\partial x_{1}}\right)^{-1} \frac{\partial h}{\partial x_{j}} .
$$

Это доказывает, что потенциал $F$ удовлетворяет уравнениям ВДВВ.

Связь между комплексами Ленарда и уравнениями ВДВВ имеет много других аспектов, которые заслуживают внимания. Упомянем два из них: в случае, когда $K_{1}=\mathrm{Id}$, и в более сложном случае, когда $K_{1} \neq \mathrm{Id}$.

Случай $K_{1}=\mathrm{Id}$. В этом случае комплекс Ленарда полностью характеризуется потенциалом $F$, причем есть взимно однозначное соответствие между комплексами Ленарда этого типа и решениями уравнений ВДВВ. Другими словами, можно легко построить комплекс Ленарда с $K_{1}=\mathrm{Id}$, начиная с решения классического уравнения ВДВВ.

Случай $K_{1} \neq \mathrm{Id}$. В этом случае потенциал $F$ не полностью характеризует комплекс Ленарда. Появляется вторая функция $G$, которая связана с $F$ парой интересных уравнений. Пусть $\left(\lambda_{1}, \ldots, \lambda_{n}\right)$ - компоненты векторного поля $X$ в $x$-координатах:

$$
X=\sum \lambda_{k} \frac{\partial}{\partial x^{k}}
$$

Тогда можно доказать, что матрица

$$
g=\sum \lambda_{k} \frac{\partial h}{\partial x_{k}}
$$

есть матрица Гессе функции $G$ и что $F$ и $G$ удовлетворяют обобщенным уравнениям ВДВВ

$$
\frac{\partial h}{\partial x_{j}} g^{-1} \frac{\partial h}{\partial x_{l}}=\frac{\partial h}{\partial x_{l}} g^{-1} \frac{\partial h}{\partial x_{j}} .
$$

Мы опустим доказательство этих уравнений, но приведем пример, который имеет отношение к нашей теме. Функция

$$
F\left(x_{1} x_{2} x_{3}\right)=\sum_{i<j}^{3}\left(x_{i}-x_{j}\right)^{2} \ln \left(x_{i}-x_{j}\right)^{2}+\frac{1}{2} \sum_{i=1}^{3} x_{i}^{2} \ln x_{i}^{2}
$$

- это одно самых простых решений уравнений ВДВВ (6) типа Веселова. Как отмечалось в разделе 2, она порождает комплекс Ленарда второго типа. Следовательно, она должна удовлетворять уравнениям (7). В самом деле, можно проверить, что матрица

$$
g=\sum_{i=1}^{3} \frac{1}{4} x_{i} \frac{\partial h}{\partial x_{i}}
$$

является матрицей Гессе функции

$$
G=\frac{3}{8}\left(x_{1}^{2}+x_{2}^{2}+x_{3}^{2}\right)-\frac{1}{4}\left(x_{1} x_{2}+x_{2} x_{3}+x_{3} x_{2}\right)
$$

и что функции $F$ и $G$ удовлетворяют обобщенным уравнениям ВДВВ (7). Последнее уравнение представляется особенно интересным. Оно подразумевает, что 
функция $F$ является также решением обычных уравнений ВДВВ. Это означает, что некоторые решения сильно нелинейных уравнений ВДВВ могут быть найдены при решении задачи только с квадратичными нелинейностями.

Еще один момент, который мы вынуждены пропустить, это связь с теорией фробениусовых многообразий. Известно, что Дубровин [5] в 90-е годы придумал красивые и перспективные концепции фробениусовых многообразий [6], [7], чтобы дать геометрическую интерпретацию уравнений ВДВВ. Понятия фробениусового многообразия и комплекса Ленарда должны быть сильно связаны. Они дают две разные точки зрения на один и тот же объект, поскольку используют разные геометрические структуры. Некоторые детали этой связи до сих пор отсутствуют.

\section{4. ЭКВИВАРИАНТНЫЕ КОМПЛЕКСЫ}

Порядок операторов рекурсии $K_{1}, K_{2}, \ldots, K_{n}$ не имеет значения, эти операторы можно менять местами друг с другом. В процессе изучения поведения комплекса Ленарда при изменении порядка операторов рекурсии предлагается ввести фиксированный класс комплексов Ленарда, которые называются эквивариантными по отношению к симметрической группе. Они обладают таким свойством, что перестановка операторов $K_{j}$ и $K_{l}$ равносильна перестановке координат $a_{j}$ и $a_{l}$.

Эквивариантные комплексы Ленарда определяются следующим образом. Пусть $S_{n}$ - симметрическая группа, действующая на $a$-координатах $\left(a_{1}, \ldots, a_{n}\right)$. Обозначим через $\sigma_{j l}=\left(a_{j}, a_{l}\right)$ транспозицию координат $a_{j}$ и $a_{l}$. Мы представляем эту перестановку как отображение $\sigma_{j l}: M \rightarrow M$. Соответственно, это действие поднимается на 1-формы и векторные поля, определенные на многообразии $M$. Поднятое действие задается обратным образом $\sigma_{j l}^{*} 1$-форм и прямым образом $\sigma_{* j l}$ векторных полей. Примеры этих действий следующие:

$$
\sigma_{j l}^{*}\left(d a_{j}\right)=d a_{l}, \quad \sigma_{j l}^{*}\left(d a_{m}\right)=d a_{m}, \quad \sigma_{* j l}\left(\frac{\partial}{\partial a_{j}}\right)=\frac{\partial}{\partial a_{l}}, \quad m \neq j, l .
$$

Наконец, обобщим действие $S_{n}$ на операторах рекурсии в соответствии с обычными правилами тензорного анализа.

ОПРЕДЕЛЕНИЕ 4. Комплекс Ленарда $\left(K_{j}, d A, X\right)$ называется эквивариантным по отношению к действию симметрической группы $S_{n}$ на а-координатах $\left(a_{1}, \ldots, a_{n}\right)$, если выполняются следующие условия:

1) векторное поле $X$ и 1-форма $d A$ инвариантны:

$$
\sigma_{j l}^{*}(d A)=d A, \quad \sigma_{* j l}(X)=X
$$

2) транспозиция $\sigma_{j l}: M \rightarrow M$ меняет операторы $K_{j}$ и $K_{l}$ :

$$
\sigma_{j l}\left(K_{j}\right)=K_{l}
$$

Разберем в явном виде условия эквивариантности при $\operatorname{dim} M=3$. Пусть перестановка $\sigma_{j l}$ действует отдельно на квадраты 1-форм. Это действие создает новый квадрат. Условие эквивариантности означает, что новый квадрат совпадает со ста- 
рым с точностью до перестановки $l$-х и $j$-х строчек и столбцов. Следовательно, для эквивариантных комплексов выполняется следующая диаграмма:

\begin{tabular}{|c|c|c|c|}
\hline$\sigma_{12}^{*}(d A)$ & $\sigma_{12}^{*}\left(d a_{1}\right)$ & $\sigma_{12}^{*}\left(d a_{2}\right)$ & $\sigma_{12}^{*}\left(d a_{3}\right)$ \\
\hline$\sigma_{12}^{*}\left(d a_{1}\right)$ & $\sigma_{12}^{*}(d P)$ & $\sigma_{12}^{*}(d Q)$ & $\sigma_{12}^{*}(d R)$ \\
\hline$\sigma_{12}^{*}\left(d a_{2}\right)$ & $\sigma_{12}^{*}(d Q)$ & $\sigma_{12}^{*}(d S)$ & $\sigma_{12}^{*}(d T)$ \\
\hline$\sigma_{12}^{*}\left(d a_{3}\right)$ & $\sigma_{12}^{*}(d R)$ & $\sigma_{12}^{*}(d T)$ & $\sigma_{12}^{*}(d V)$
\end{tabular}$=$\begin{tabular}{|c|c|c|c|}
\hline$d A$ & $d a_{2}$ & $d a_{1}$ & $d a_{3}$ \\
\hline$d a_{2}$ & $d S$ & $d Q$ & $d T$ \\
\hline$d a_{1}$ & $d Q$ & $d P$ & $d R$ \\
\hline$d a_{3}$ & $d T$ & $d R$ & $d a_{3}$ \\
\hline
\end{tabular}

Эта диаграмма показывает, что 1-формы эквивариантного комплекса удовлетворяют условиям симметрии

$$
\sigma_{12}^{*}(d A)=d A, \quad \sigma_{12}^{*}(d P)=d S, \quad \sigma_{12}^{*}(d Q)=d Q, \quad \sigma_{12}^{*}(d R)=d T .
$$

Таким образом, можно понять, что эквивариантный квадрат 1-форм полностью определяется тремя 1-формами: центральной 1-формой $d A, 1$-формой $d P$ на диагонали квадрата и 1-формой $d Q$ вне диагонали. Остальные 1-формы восстанавливаются в соответствии с соотношениями

$$
\sigma_{12}^{*}(d P)=d S, \quad \sigma_{13}^{*}(d P)=d V, \quad \sigma_{23}^{*}(d Q)=d R, \quad \sigma_{13}^{*}(d Q)=d T .
$$

Эта операция проясняется с помощью следующего примера.

ПримеР 3. По причинам, которые приводятся ниже в разделе 5 , выберем исходные 1-формы $(d A, d P, d Q)$ следующим образом:

$$
\begin{aligned}
d A= & \left(2 a_{1}+a_{2}+a_{3}\right) d a_{1}+\left(a_{1}+2 a_{2}+a_{3}\right) d a_{2}+\left(a_{1}+a_{2}+2 a_{3}\right) d a_{3}, \\
d Q=- & \frac{1}{4} \frac{d\left(a_{1}-a_{2}\right)}{a_{1}-a_{2}}, \\
d P= & \frac{6 a_{1}^{2}-2 a_{1} a_{2}-2 a_{2} a_{3}-a_{2}^{2}-a_{3}^{2}}{4\left(2 a_{1}+a_{2}+a_{3}\right)\left(a_{1}-a_{2}\right)\left(a_{1}-a_{3}\right)} d a_{1}-\frac{2 a_{2}+a_{1}+a_{3}}{4\left(2 a_{1}+a_{2}+a_{3}\right)\left(a_{1}-a_{2}\right)} d a_{2}+ \\
& \quad+\frac{2 a_{3}+a_{1}+a_{2}}{4\left(2 a_{1}+a_{2}+a_{3}\right)\left(a_{1}-a_{3}\right)} d a_{3},
\end{aligned}
$$

В случае действия симметрической группы $S_{3}$ на координатах $\left(a_{1}, a_{2}, a_{3}\right)$ сначала построим орбиту, содержащую $d Q$, получим

$$
d Q=-\frac{1}{4} \frac{d\left(a_{1}-a_{2}\right)}{a_{1}-a_{2}}, \quad d R=-\frac{1}{4} \frac{d\left(a_{1}-a_{3}\right)}{a_{1}-a_{3}}, \quad d T=-\frac{1}{4} \frac{d\left(a_{2}-a_{3}\right)}{a_{2}-a_{3}} .
$$

Аналогично для орбиты, содержащей $d P$, получим

$$
\begin{aligned}
d P= & \frac{6 a_{1}^{2}-2 a_{1} a_{2}-2 a_{2} a_{3}-a_{2}^{2}-a_{3}^{2}}{4\left(2 a_{1}+a_{2}+a_{3}\right)\left(a_{1}-a_{2}\right)\left(a_{1}-a_{3}\right)} d a_{1}-\frac{2 a_{2}+a_{1}+a_{3}}{4\left(2 a_{1}+a_{2}+a_{3}\right)\left(a_{1}-a_{2}\right)} d a_{2}+ \\
& +\frac{2 a_{3}+a_{1}+a_{2}}{4\left(2 a_{1}+a_{2}+a_{3}\right)\left(a_{1}-a_{3}\right)} d a_{3}, \\
d S=- & \frac{2 a_{1}+a_{2}+a_{3}}{4\left(2 a_{2}+a_{1}+a_{3}\right)\left(a_{2}-a_{1}\right)} d a_{1}+\frac{6 a_{2}^{2}-2 a_{2} a_{3}-2 a_{2} a_{1}-a_{3}^{2}-a_{1}^{2}}{4\left(2 a_{2}+a_{1}+a_{3}\right)\left(a_{2}-a_{1}\right)\left(a_{2}-a_{3}\right)} d a_{2}- \\
& \quad-\frac{2 a_{3}+a_{2}+a_{1}}{4\left(2 a_{2}+a_{1}+a_{3}\right)\left(a_{2}-a_{3}\right)} d a_{3},
\end{aligned}
$$




$$
\begin{aligned}
d V=- & \frac{2 a_{1}+a_{2}+a_{3}}{4\left(2 a_{3}+a_{2}+a_{1}\right)\left(a_{3}-a_{1}\right)} d a_{1}-\frac{2 a_{2}+a_{1}+a_{3}}{4\left(2 a_{3}+a_{2}+a_{1}\right)\left(a_{3}-a_{2}\right)} d a_{2}+ \\
& +\frac{6 a_{3}^{2}-2 a_{1} a_{3}-2 a_{2} a_{3}-a_{1}^{2}-a_{3}^{2}}{4\left(2 a_{3}+a_{2}+a_{1}\right)\left(a_{3}-a_{1}\right)\left(a_{3}-a_{2}\right)} d a_{3} .
\end{aligned}
$$

Таким способом квадрат заполнен с использованием эквивариантности. Далее мы используем квадрат 1-форм, чтобы определить операторы рекурсии $\left(K_{1}, K_{2}, K_{3}\right)$ :

$$
\begin{array}{lll}
K_{1}\left(d a_{1}\right)=d P, & K_{1}\left(d a_{2}\right)=d Q, & K_{1}\left(d a_{3}\right)=d R, \\
K_{2}\left(d a_{1}\right)=d Q, & K_{2}\left(d a_{2}\right)=d S, & K_{2}\left(d a_{3}\right)=d T, \\
K_{3}\left(d a_{1}\right)=d R, & K_{3}\left(d a_{2}\right)=d T, & K_{3}\left(d a_{3}\right)=d V .
\end{array}
$$

Чтобы завершить построение комплекса Ленарда, нужно определить векторное поле $X$. По причинам, объясненным ниже (см. раздел 5), выберем векторное поле в виде

$$
X=a_{1} \frac{\partial}{\partial a_{1}}+a_{2} \frac{\partial}{\partial a_{2}}+a_{3} \frac{\partial}{\partial a_{3}} .
$$

Получим следующие векторные поля $\left(K_{1} X, K_{2} X, K_{3} X\right)$ :

$$
\begin{aligned}
& X_{1}=\frac{1}{4}\left(+3 \frac{\partial}{\partial a_{1}}-\frac{\partial}{\partial a_{2}}-\frac{\partial}{\partial a_{3}}\right), \\
& X_{2}=\frac{1}{4}\left(-\frac{\partial}{\partial a_{1}}+3 \frac{\partial}{\partial a_{2}}-\frac{\partial}{\partial a_{3}}\right), \\
& X_{3}=\frac{1}{4}\left(-\frac{\partial}{\partial a_{1}}-\frac{\partial}{\partial a_{2}}+3 \frac{\partial}{\partial a_{3}}\right) .
\end{aligned}
$$

Они коммутируют, а следовательно, они определяют $x$-координаты. Такие координаты связаны с $a$-координатами с помощью соотношений

$$
x_{1}=2 a_{1}+a_{2}+a_{3}, \quad x_{2}=2 a_{2}+a_{3}+a_{1}, \quad x_{3}=2 a_{3}+a_{1}+a_{2} .
$$

Можно проверить, что построенный комплекс удовлетворяет условию, наложенному на комплекс Ленарда. Соответственно, согласно предложению 1 он определяет решение уравнений ВДВВ. Это решение можно найти при помощи записи квадрата 1-форм в $x$-координатах. Можно проверить тождества $d Q=d F_{12}, d R=d F_{13}$, $d T=d F_{23}, d P=d F_{11}, d S=d F_{22}, d V=d F_{33}$, где $F_{j k}$ - вторые частные производные функции

$$
F\left(x_{1} x_{2} x_{3}\right)=\frac{1}{16} \sum_{i<j}^{3}\left(x_{i}-x_{j}\right)^{2} \ln \left(x_{i}-x_{j}\right)^{2}+\frac{1}{16} \sum_{i=1}^{3} x_{i}^{2} \ln x_{i}^{2}
$$

по координатам $x_{j}$ и $x_{k}$. Это вычисление показывает, что самое простое решение типа Веселова уравнений ВДВВ - потенциал эквивариантного комплекса Ленарда.

\section{5. СИСТЕМЫ ВЕСЕЛОВА}

Требование эквивариантности, состоящее в наложении условий симметрии, значительно уменьшает количество свободных параметров, которые используются для построения комплекса Ленарда. В то же время это также уменьшает число независимых условий, которым должны удовлетворять параметры. Цель данного раздела - показать, что баланс между уменьшением числа степеней свободы и уменьшением числа условий является подходящим. Это обстоятельство позволит построить 
семейство эквивариантных комплексов Ленарда на $\mathbb{R}^{3}$, зависящих от одного параметра.

Главная идея заключается в том, что уравнения, определяющие комплекс Ленарда, могут быть решены чисто алгебраическим образом в классе эквивариантных дифференциальных форм логарифмического типа, т. е. в классе 1-форм типа

$$
d Q=\sum_{k=1}^{m} \sigma_{k} \frac{d B_{k}}{B_{k}}
$$

где $\sigma_{k}$ - параметры, а $B_{k}$ - функции, выбранные соответствующим образом. Было бы полезно объяснить причину выбора этого класса форм посредством более тщательного рассмотрения структуры условий, определяющих комплекс Ленарда. Для краткости, однако, приведем конечный результат.

Пусть

$$
A=\frac{1}{2} \alpha\left(a_{1}^{2}+a_{2}^{2}+a_{3}^{2}\right)+\beta\left(a_{1} a_{2}+a_{2} a_{3}+a_{3} a_{2}\right)
$$

- наиболее общий квадратичный полином на $\mathbb{R}^{3}$, который инвариантен по отношению к действию симметрической группы $S_{3}$. Предположим, что $(\alpha-\beta)^{2}(2 \beta+\alpha) \neq 0$, тогда матрица Гессе функции $A$ обратима. Заметим, что частные производные $A$

$$
A_{1}=\alpha a_{1}+\beta\left(a_{2}+a_{3}\right), \quad A_{2}=\alpha a_{2}+\beta\left(a_{3}+a_{1}\right), \quad A_{3}=\alpha a_{3}+\beta\left(a_{1}+a_{2}\right)
$$

образуют в этом случае новую систему координат на $\mathbb{R}^{3}$. Очевидно, что перестановка $\sigma_{j l}$ действует так же, как она действовала на $a$-координатах. В новых координатах определим пару 1-форм

$$
d Q=\sum_{k=1}^{m} \sigma_{k}\left(\frac{d\left(A_{1}+\eta_{k} A_{2}\right)}{A_{1}+\eta_{k} A_{2}}+\frac{d\left(A_{2}+\eta_{k} A_{1}\right)}{A_{2}+\eta_{k} A_{1}}\right)
$$

И

$$
\begin{gathered}
d P=\sigma_{0} \frac{d A_{1}}{A_{1}}+\sum_{k=1}^{m} \frac{\sigma_{k}}{\eta_{k}}\left(\frac{d\left(A_{1}+\eta_{k} A_{2}\right)}{A_{1}+\eta_{k} A_{2}}+\frac{d\left(A_{1}+\eta_{k} A_{3}\right)}{A_{1}+\eta_{k} A_{3}}\right)+ \\
+\sum_{k=1}^{m} \sigma_{k} \eta_{k}\left(\frac{d\left(A_{2}+\eta_{k} A_{1}\right)}{A_{2}+\eta_{k} A_{1}}+\frac{d\left(A_{3}+\eta_{k} A_{1}\right)}{A_{3}+\eta_{k} A_{1}}\right)
\end{gathered}
$$

где $\left(\sigma_{0}, \sigma_{k}, \eta_{k}\right)-2 m+1$ параметров. Предполагается, что они подчиняются условиям

$$
\begin{aligned}
2 \sum_{k=1}^{m} \sigma_{k} & =\frac{\beta}{(\beta-\alpha)(2 \beta+\alpha)}, \\
\sigma_{0}+2 \sum_{k=1}^{m}\left(\frac{\sigma_{k}}{\eta_{k}}+\sigma_{k} \eta_{k}\right) & =\frac{\alpha+\beta}{(\alpha-\beta)(2 \beta+\alpha)} .
\end{aligned}
$$

Указанные выше 1-формы явно удовлетворяют условиям симметрии

$$
\sigma_{12}^{*}(d Q)=d Q, \quad \sigma_{23}^{*}(d P)=d P,
$$

и, следовательно, их можно использовать для построения эквивариантного квадрата 1-форм, следуя процедуре, описанной в разделе 4. Обозначим, как и раньше, 
через $d R=\sigma_{23}^{*}(d Q), d T=\sigma_{13}^{*}(d Q)$ элементы орбиты симметрической группы, содержащей $d Q$, и через $d S=\sigma_{12}^{*}(d P), d V=\sigma_{13}^{*}(d P)$ - элементы орбиты симметрической группы, содержащей $d P$. Далее используем шесть 1-форм $(d Q, d R, d T, d P, d S, d V)$, чтобы определить операторы рекурсии $\left(K_{1}, K_{2}, K_{3}\right)$ в соответствии со стандартными соотношениями (в $a$-координатах):

$$
\begin{array}{lll}
K_{1}\left(d a_{1}\right)=d P, & K_{2}\left(d a_{1}\right)=d Q, & K_{3}\left(d a_{1}\right)=d R, \\
K_{1}\left(d a_{2}\right)=d Q, & K_{2}\left(d a_{2}\right)=d S, & K_{3}\left(d a_{2}\right)=d T, \\
K_{1}\left(d a_{3}\right)=d R, & K_{2}\left(d a_{3}\right)=d T, & K_{3}\left(d a_{3}\right)=d V .
\end{array}
$$

Наконец, рассмотрим векторное поле

$$
X=A_{1} \frac{\partial}{\partial A_{1}}+A_{2} \frac{\partial}{\partial A_{2}}+A_{3} \frac{\partial}{\partial A_{3}},
$$

и пусть

$$
X_{1}=\frac{\partial}{\partial A_{1}}, \quad X_{2}=\frac{\partial}{\partial A_{2}}, \quad X_{3}=\frac{\partial}{\partial A_{3}} .
$$

ПреДЛОЖЕНИЕ 2. Тензорнъе поля $\left(K_{j}, d A, X\right)$, указанные выше, определяют эквивариантный комплекс Ленарда, если параметры $\left(\sigma_{k}, \eta_{k}\right)$ выбраны таким образом, что 1-форма $K_{3} d Q$ удовлетворяет условию симметрии

$$
\sigma_{23}^{*}\left(K_{3} d Q\right)=K_{3} d Q
$$

под действием транспозиции координат $A_{2}$ и $A_{3}$. Следовательно, согласно предложению 1 каждое решение этого уравнения определяет решение уравнений ВДВВ в $\mathbb{R}^{3}$.

ДокАзАтельство. Надо показать, что указанные выше тензорные поля удовлетворяют четырем условиям:

1) $K_{j}(d A)=d a_{j}$

2) $K_{j}(X)=X_{j}$

3) $K_{j} K_{l}(d A)=d A_{j l}$;

4) $K_{j} K_{l}-K_{l} K_{j}=0$.

Первое условие означает, что $\left(K_{j}, d A\right)$ определяет цепь точных 1-форм. Из второго условия следует, что $\left(K_{j}, X\right)$ определяет цепь коммутирующих векторных полей. Третье условие означает, что $\left(K_{j}, d A\right)$ определяет квадрат точных 1-форм. Наконец, четвертое условие говорит о том, что квадрат 1-форм симметричен. Без этого условия матричный потенциал комплекса Ленарда не может быть матрицей Гессе функции $F$, которая удовлетворяет уравнениям ВДВВ. В соответствии с этими четырьмя условиями мы разделим доказательство на четыре части.

1. Цепь 1-форм. Рассмотрим условие 1: $K_{1}(d A)=d a_{1}$. Записав $d A$ в базисе $d a_{j}$, перепишем условие в эквивалентной форме

$$
A_{1} d P+A_{2} d Q+A_{3} d R=d a_{1}
$$

с учетом определения $K_{1}$. Подставляя в это уравнение определения 1 -форм $d P, d Q$ и $d R$, можно увидеть, что знаменатели сокращаются и что условие 1 упрощается до линейного уравнения

$$
\begin{aligned}
\sigma_{0} d A_{1} & +\sum_{k} \frac{\sigma_{k}}{\eta_{k}}\left[d\left(A_{1}+\eta_{k} A_{2}\right)+d\left(A_{1}+\eta_{k} A_{3}\right)\right]+ \\
& +\sum_{k} \sigma_{k}\left[d\left(A_{2}+\eta_{k} A_{1}\right)+d\left(A_{3}+\eta_{k} A_{1}\right)\right]=d a_{1} .
\end{aligned}
$$


Это и есть глубокая причина такого специфического выбора 1-форм $d P$ и $d Q$. Уравнение теперь интегрируемо, и его справедливость следует из (12). Остальные условия $K_{2}(d A)=d a_{2}$ и $K_{3}(d A)=d a_{3}$ следуют из эквивариантности.

2. Цепь векторных полей. Рассмотрим векторное поле $K_{1}(X)$ на базисе $d a_{j}$, находим

$$
\begin{aligned}
d a_{1}\left(K_{1} X\right) & =d P(X)=\sigma_{0}+2 \sum_{k}\left(\frac{\sigma_{k}}{\eta_{k}}+\sigma_{k} \eta_{k}\right)= \\
& =\frac{\alpha+\beta}{(\alpha-\beta)(2 \beta+\alpha)}=d a_{1}\left(X_{1}\right) .
\end{aligned}
$$

Также находим

$$
\begin{aligned}
& d a_{2}\left(K_{1} X\right)=2 \sum_{k} \sigma_{k}=\frac{\beta}{(\beta-\alpha)(2 \beta+\alpha)}=d a_{2}\left(X_{1}\right), \\
& d a_{3}\left(K_{1} X\right)=2 \sum_{k} \sigma_{k}=\frac{\beta}{(\beta-\alpha)(2 \beta+\alpha)}=d a_{3}\left(X_{1}\right) .
\end{aligned}
$$

Вместе эти тождества показывают, что $K_{1}(X)=X_{1}$, что и требовалось доказать. Остальные два условия $K_{2}(X)=X_{2}$ и $K_{3}(X)=X_{3}$ следуют из эквивариантности.

3. Коммутирование операторов рекурсии. Рассмотрим коммутатор $\left[K_{2}, K_{1}\right]$ на базисе $d a_{j}$, получим

$$
\begin{aligned}
& {\left[K_{2}, K_{1}\right]\left(d a_{1}\right)=K_{2} d P-K_{1} d Q,} \\
& {\left[K_{2}, K_{1}\right]\left(d a_{2}\right)=K_{2} d Q-K_{1} d S,} \\
& {\left[K_{2}, K_{1}\right]\left(d a_{3}\right)=K_{2} d R-K_{1} d T}
\end{aligned}
$$

в силу определения операторов рекурсии. Заметим, что второе соотношение следует из первого в силу эквивариантности. Условия на коммутаторы $\left[K_{3}, K_{1}\right]$ и $\left[K_{3}, K_{2}\right]$ следуют из предыдущих также в силу эквивариантности. Таким образом, осталась только пара коммутационных соотношений

$$
\begin{aligned}
& K_{2} d P=K_{1} d Q, \\
& K_{2} d R=K_{1} d T .
\end{aligned}
$$

Заметим, что соотношение (15) следует из предположения (13). Действительно, в силу эквивариантности

$$
\sigma_{23}\left(K_{3} d Q\right)=K_{2} \sigma_{23}(d Q)=K_{2} d R,
$$

и, таким образом, из предположения (13) следует

$$
K_{2} d R=K_{3} d Q
$$

Тогда соотношение (15) следует из эквивариантности:

$$
K_{2} d R=\sigma_{13}\left(K_{2} d R\right)=\sigma_{13}\left(K_{3} d Q\right)=K_{1} \sigma_{13}(d Q)=K_{1} d T .
$$

Наконец, заметим, что условие (14) следует из (13). Действительно, условие (14) эквивалентно

$$
A_{1} K_{2} d P=A_{1} K_{1} d Q
$$


Так как

$$
A_{1} d P+A_{2} d Q+A_{3} d R=d a_{1}
$$

получаем

$$
A_{1} K_{2} d P=-\left(A_{2} K_{2} d Q+A_{3} K_{2} d R\right)+K_{2} d a_{1}=-\left(A_{2} K_{2} d Q+A_{3} K_{3} d Q\right)+d Q .
$$

Таким образом, осталось доказать, что

$$
A_{1} K_{1}(d Q)+A_{2} K_{2}(d Q)+A_{3} K_{3}(d Q)=d Q
$$

Это верно, так как

$$
A_{1} K_{1}+A_{2} K_{2}+A_{3} K_{3}=\mathrm{Id} .
$$

Действительно,

$$
\left(A_{1} K_{1}+A_{2} K_{2}+A_{3} K_{3}\right) d a_{1}=A_{1} d P+A_{2} d Q+A_{3} d R=d a_{1}
$$

и аналогично для $a_{2}$ и $a_{3}$. Таким образом, доказано, что операторы рекурсии коммутируют, если справедливо предположение (13).

4. Квадрат 1-форм. Соотношения $K_{j} K_{l}(d A)=d A_{j l}$ являются простым следствием определения операторов рекурсии.

Возможность построения эквивариантных комплексов Ленарда на $\mathbb{R}^{3}$ (и, вероятно, на $\mathbb{R}^{n}$ ) зависит от возможности решения условия (13). Это очень строгое ограничение, поскольку неизвестными являются параметры, в то время как ограничение зависит явным образом (нелинейным и сложным образом) от координат. В некотором смысле удивительно, что у него есть решения. Здесь мы описываем решения условия симметрии (13) в классе форм логарифмического типа, рассматриваемого в настоящем разделе.

Решение относится к случаю $m=2$, где 1-формы комплекса Ленарда зависят от семи параметров $\left(\alpha, \beta ; \sigma_{0}, \sigma_{1}, \sigma_{2} ; \eta_{1}, \eta_{2}\right)$. Два из них $-\sigma_{0}$ и $\sigma_{1}-$ связаны соотношениями (12). Другие параметры могут быть выбраны так, чтобы удовлетворять условию симметрии (13) в общей точке $\left(A_{1}, A_{2}, A_{3}\right)$. Оказывается, что выбор параметров $\left(\eta_{1}, \eta_{2}\right)$ имеет важное значение для достижения этой цели. Действительно, если положить

$$
\eta_{1}=+1, \quad \eta_{2}=-1,
$$

можно увидеть, что 1 -форма $\sigma_{23}^{*}\left(K_{3} d Q\right)-K_{3} d Q$ примет хороший факторизованный вид

$$
\sigma_{23}^{*}\left(K_{3} d Q\right)-K_{3} d Q=\Phi\left(\alpha, \beta, \sigma_{2}\right) \Psi\left(A_{1}, A_{2}, A_{3}\right)\left(\frac{d A_{3}}{A_{3}}-\frac{d A_{2}}{A_{2}}\right),
$$

где

$$
\begin{gathered}
\Psi\left(A_{1}, A_{2}, A_{3}\right)=\frac{A_{1} A_{2}+A_{2} A_{3}+A_{3} A_{1}}{\left(A_{1}+A_{2}\right)\left(A_{2}+A_{3}\right)\left(A_{3}+A_{1}\right)}, \\
\Phi\left(\alpha, \beta, \sigma_{2}\right)=\frac{2 \beta\left(2 \alpha \beta \sigma_{2}+2 \alpha^{2} \sigma_{2}-4 \beta^{2} \sigma_{2}+\beta\right)\left(8 \alpha \beta \sigma_{2}+8 \alpha^{2} \sigma_{2}-16 \beta^{2} \sigma_{2}+\alpha+3 \beta\right)}{(\alpha-\beta)^{2}(2 \beta+\alpha)^{2}} .
\end{gathered}
$$

Следовательно, условию симметричности (13) удовлетворяет любой выбор параметров $\left(\alpha, \beta, \sigma_{2}\right)$ таких, что

$$
\Phi\left(\alpha, \beta, \sigma_{2}\right)=0 .
$$


Есть три возможных варианта, каждый оставляет два параметра свободными. Тем не менее один параметр всегда может быть перемасштабирован. Таким образом, конечный комплекс Ленарда эффективно зависит только от одного свободного параметра.

Случай $m=2$ является базовым. Мы полагаем, что общий случай $m>2$ можно свести к случаю $m=2$. Действительно, условие симметрии (13) достаточно строгое для того, чтобы из него следовало условие $\eta_{k}= \pm 1$. По этой причине кажется вероятным, что не должно быть других эквивариантных комплексов на $\mathbb{R}^{3}$, кроме уже представленного. Это однопараметрическое семейство эквивариантных комплексов Ленарда, однако, является достаточно общим, по крайней мере оно включает решения Веселова типа $A_{3}$ и $B_{3}$.

\section{6. ЗАКЛЮЧИТЕЛЬНЫЕ ЗАМЕЧАНИЯ}

В этой статье мы попытались показать, что геометрическое представление комплексов Ленарда является удобным переходом к решениям уравнений ВДВВ. Мы также показали, что понятие эквивариантности позволяет свести построение эквивариантных комплексов Ленарда на $\mathbb{R}^{3}$ к алгебраической проблеме, точнее к решению условия симметрии

$$
\sigma_{23}\left(K_{3} d Q\right)=K_{3} d Q
$$

в классе 1-форм логарифмического типа. Наконец, показано, что это условие всегда допускает однопараметрическое семейство решений на $\mathbb{R}^{3}$. Каждое из них индуцирует решение уравнений ВДВВ. Вопрос о том, сколько решений Веселова содержатся в этом однопараметрическом семействе эквивариантных комплексов, до сих пор не решен. Остается неясным, все ли комплексы Ленарда, связанные с решениями Веселова согласно предложению 1, являются эквивариантными.

Благодарности. Автор особенно благодарен Б. Конопельченко за его дружескую поддержку во время исследования уравнений ВДВВ, а также благодарен П. Лорезони и А. Раймондо за полезные обсуждения систем Веселова типа $A_{3}$ и $B_{3}$.

\section{Список литературы}

[1] A. P. Veselov, Phys. Lett. A, 261:5-6 (1999), 267-302.

[2] M.V. Feigin, A.P. Veselov, "On the geometry of V-system", Geometry, Topology, and Mathematical Physics. S. P. Novikov's seminar: 2006-2007, American Mathematical Society Translations. Ser. 2., 224, eds. V. M. Buchstaber, I. M. Krichever, AMS, Providence, RI, 2008, 111-123.

[3] F. Magri, SIGMA, 8 (2012), 076, 7 pp., arXiv: 1210.5320.

[4] S.P. Tsarev, "Integrability of equations of hydrodynamic type from the end of the 19th to the end of the 20th century", Integrability: the Seiberg-Witten and Whitham equations (Edinburgh, Scotland, UK, 14-19 September, 1998), eds. H. W. Braden, I. M. Krichever, Gordon and Breach, Amsterdam, 2000, 251-265.

[5] B. Dubrovin, "Geometry of 2D topological field theory", Integrable Systems and Quantum Groups, Lectures given at the 1st Session of the Centro Internazionale Matematico Estivo (C.I.M.E.) (Montecatini Terme, Italy, June 14-22, 1993), Lecture Notes in Mathematics, 1620, eds. M. Francaviglia, S. Greco, Springer, Berlin, 1996, 120-348.

[6] Y. Manin, Frobenius Manifolds, Quantum Cohomology, and Moduli Spaces, American Mathematical Society Colloquium Publications, 47, AMS, Providence, RI, 1999.

[7] C. Hertling, Frobenius Manifolds and Moduli Spaces for Singularities, Cambridge Tracts in Mathematics, 151, Cambridge Univ. Press, Cambridge, 2002. 